\title{
Dataset of Vietnamese students' intention in respect of study abroad before and during COVID-19 pandemic
}

\section{Authors}

Hiep-Hung Pham ${ }^{1}$, Quang-Anh Phan ${ }^{2}$, Anh-Duc Hoang ${ }^{3}$, Yen-Chi Nguyen ${ }^{3}$, Ngoc-Thuy Ta ${ }^{3}$, Viet-Hung Dinh ${ }^{4}$

\section{Affiliations}

1. Phu Xuan University, Hue 49000, Vietnam

2. PostDoc at Department of Social Anthropology and Cultural Studies, University of Zurich

3. EdLab Asia Educational Research and Development Centre, Hanoi 100000, Vietnam

4. University of Labour and Social Affairs, Hanoi 100000, Vietnam

\section{Corresponding author(s)}

Hiep-Hung Pham (phamhunghiep@pxu.edu.vn)

\begin{abstract}
The Covid-19 Pandemic had completely disrupted the worldwide educational system. Many schools chose the online delivery mode for students in case learning losses incurred during social distance decree. However, as to these students who are currently in the study abroad planning stages, reached an intention crossroads, whether standing for certain unchanging decisions in study abroad destinations or changing swiftly due to the unexpected policies in quarantine. This case opened to interpretation, which was based on our e-survey since 3 May to 13 May 2020 with 397 responses covering a range of Vietnamese students. In this dataset, we focused on (i) Students' Demographics; (ii) The previous intention of students to study abroad before and during the Covid19 ravaged and (iii) Their intention afterwards.
\end{abstract}

\section{Keywords}

Education; Education system; Study abroad; COVID-19; Vietnam

Specifications Table

\begin{tabular}{|l|l|}
\hline Subject & Education, Education Management \\
\hline $\begin{array}{l}\text { Specific } \\
\text { subject area }\end{array}$ & Study abroad \\
\hline Type of data & Raw data in excel file, and analysed data \\
\hline $\begin{array}{l}\text { How data were } \\
\text { acquired }\end{array}$ & $\begin{array}{l}\text { Data was gathered using an online survey and converted into the .xlsx format } \\
\text { for formal analysis in SPSS v.20. }\end{array}$ \\
\hline
\end{tabular}




\begin{tabular}{|l|l|}
\hline Data format & $\begin{array}{l}\text { Raw } \\
\text { Analyzed }\end{array}$ \\
\hline $\begin{array}{l}\text { Parameters for } \\
\text { data collection }\end{array}$ & $\begin{array}{l}\text { This research focuses on Vietnamese students (high school level and tertiary } \\
\text { level) who are going to plan for study abroad. }\end{array}$ \\
\hline $\begin{array}{l}\text { Description of } \\
\text { data collection }\end{array}$ & $\begin{array}{l}\text { An online survey has been delivered to random students throughout some } \\
\text { groups related to study abroad on Facebook. From the connection within } \\
\text { group members, the survey drew } 410 \text { responses. }\end{array}$ \\
\hline $\begin{array}{l}\text { Data source } \\
\text { location }\end{array}$ & $\begin{array}{l}\text { Information is collected from student in Vietnam (Latitude } 16^{\circ} 0^{\prime} \mathrm{N}, \\
\left.\text { Longitude } 106^{\circ} 0{ }^{\prime} \mathrm{E}\right)\end{array}$ \\
\hline $\begin{array}{l}\text { Data } \\
\text { accessibility }\end{array}$ & $\begin{array}{l}\text { Repository name: Mendeley Data } \\
\text { Data identification number: } \\
\text { Direct URL to data: https://data.mendeley.com/datasets/7gpkx } 5 \mathrm{p} 3 \mathrm{z} 9 / 1\end{array}$ \\
\hline
\end{tabular}

\section{Value of the Data}

- The data acquisition makes a special contribution towards research in the field of study abroad in the worldwide pandemic context.

- The dataset can be used for additional analysis of students' intention to choose study abroad destinations due to the policy responses within the Covid-19.

- The dataset can be applied to build models related to investigate the situational factors behind students' intention to study abroad.

- The dataset conveys a message to policymakers in the education sector in respect of exporting and importing study abroad.

- The significant change in the study abroad decision of students before and during Covid19 partly contributes to overall educational models from the pandemic's damage.

\section{Data Description}

For years, study abroad brings about many benefits for students, such as personal growth, intercultural development, education and career attainment, and so on [1]. In addition, it is also a crucial source of income for the host colleges, universities and the host government $[2,3]$. In Vietnam, education is considered more of an imported service than an exported one [4], and this trend of study abroad has become more popular recently. From the year 2010 to 2019, Vietnam climbed from the ninth to the sixth place among countries with most students studying in the United States [5]. Moreover, the number of Vietnamese students studying in other countries also increased sharply, reached top 5 in Canada, top 4 in Australia, and top 2 in Japan in 2018 [6].

Since the expansion of the Coronavirus, countries around the world have taken many measures to avoid outsiders, in particular imposing travel restrictions, locking down airports, and closing borders [7]. Many teachers, including local as well as expat teachers, met so many problems. Students were suddenly acquainted with online learning. In this particular dataset we portrayed the 
decisions and factors related to Vietnamese students' study abroad before and after the COVID19. It is an extension to our recent studies related to students' learning habit during the COVID19 [10].

The dataset includes three main parts: (i) Demographic of the partakers; (ii) Factors that influence students' decision to study abroad before COVID-19; and (iii) Factors that influence students' decision to study abroad after COVID-19. The full version of the survey, variable codes and measurements can be found on Mendeley data [11]. From this dataset, researchers can study trends among Vietnamese students as well as various factors that affect students' decision of study abroad, ranging from finance, culture, to communication related.

Table 1. Descriptive statistics of participant's demographics

\begin{tabular}{|c|c|c|c|c|c|}
\hline & & Frequency & Percent & $\begin{array}{c}\text { Valid } \\
\text { Percent }\end{array}$ & $\begin{array}{c}\text { Cumulative } \\
\text { Percent }\end{array}$ \\
\hline \multirow{2}{*}{ Gender } & Male & 188 & 47.4 & 47.4 & 47.4 \\
\hline & Female & 209 & 52.6 & 52.6 & 100.0 \\
\hline \multirow{3}{*}{ Major } & $\begin{array}{l}\text { Natural science, } \\
\text { Engineering, Technology }\end{array}$ & 85 & 21.4 & 21.4 & 21.4 \\
\hline & $\begin{array}{l}\text { Social science, } \\
\text { Humanities, Art, } \\
\text { Economics, Management, } \\
\text { Business }\end{array}$ & 214 & 53.9 & 53.9 & 75.3 \\
\hline & Other & 98 & 24.7 & 24.7 & 100.0 \\
\hline \multirow{3}{*}{ Cur_Location } & Hanoi & 144 & 36.3 & 36.3 & 36.3 \\
\hline & Ho Chi Minh & 128 & 32.2 & 32.2 & 68.5 \\
\hline & Other & 125 & 31.5 & 31.5 & 100.0 \\
\hline \multirow{5}{*}{ Prior_Plan } & Asia & 42 & 10.6 & 10.6 & 10.6 \\
\hline & Europe & 53 & 13.4 & 13.4 & 23.9 \\
\hline & America & 43 & 10.8 & 10.8 & 34.8 \\
\hline & Oceania & 20 & 5.0 & 5.0 & 39.8 \\
\hline & Other & 239 & 60.2 & 60.2 & 100.0 \\
\hline \multirow{2}{*}{ Prior_Fin } & Full scholarship & 235 & 59.2 & 59.2 & 59.2 \\
\hline & Self-finance & 162 & 40.8 & 40.8 & 100.0 \\
\hline Prior_program & High school & 15 & 3.8 & 3.8 & 3.8 \\
\hline
\end{tabular}




\begin{tabular}{|l|l|c|c|c|c|}
\hline \multirow{5}{*}{} & Bachelor & 177 & 44.6 & 44.6 & 48.4 \\
\cline { 2 - 6 } & Master & 185 & 46.6 & 46.6 & 95.0 \\
\cline { 2 - 6 } & Doctor & 14 & 3.5 & 3.5 & 98.5 \\
\cline { 2 - 6 } & Other & 6 & 1.5 & 1.5 & 100.0 \\
\hline \multirow{5}{*}{ Post_Plan } & Asia & 20 & 5.0 & 5.0 & 5.0 \\
\cline { 2 - 6 } & Europe & 15 & 3.8 & 3.8 & 8.8 \\
\cline { 2 - 6 } & America & 9 & 2.3 & 2.3 & 11.1 \\
\cline { 2 - 6 } & Oceania & 12 & 3.0 & 3.0 & 14.1 \\
\cline { 2 - 6 } & Other & 57 & 14.4 & 14.4 & 28.5 \\
\cline { 2 - 6 } & Same country as & 284 & 71.5 & 71.5 & 100.0 \\
\cline { 2 - 6 } & Prior_Plan & 397 & 100.0 & 100.0 & \\
\cline { 2 - 5 } & Total & & & \\
\hline
\end{tabular}

Table 2. Descriptive statistics of various factors influencing study abroad decision

\begin{tabular}{ll|l|r|l} 
& \multirow{2}{*}{$\mathrm{N}$} & \multicolumn{2}{|c|}{ Mean } & \multicolumn{1}{l}{ S.D } \\
\hline Prior_living & 397 & 3.34 & .028 & .566 \\
\hline Prior_travel & 397 & 2.46 & .035 & .690 \\
\hline Prior_language & 397 & 3.25 & .035 & .695 \\
\hline Prior_quality & 397 & 3.67 & .027 & .536 \\
\hline Prior_degree & 397 & 3.41 & .032 & .632 \\
\hline Prior_IntlStu & 397 & 2.73 & .040 & .804 \\
\hline Prior_VnStu & 397 & 2.33 & .038 & .755 \\
\hline Prior_tuition & 397 & 3.52 & .034 & .684 \\
\hline Prior_scholarship & 397 & 3.68 & .029 & .583 \\
\hline Prior_livingcost & 397 & 3.54 & .031 & .617 \\
\hline Prior_parttimejob & 397 & 3.20 & .037 & .739 \\
\hline Prior_job & 397 & 3.55 & .033 & .655 \\
\hline Prior_immigration & 397 & 2.78 & .044 & .881 \\
\hline Prior_shortcut & 397 & 2.58 & .042 & .845 \\
\hline Prior_distance & 397 & 2.12 & .041 & .823 \\
\hline Prior_HE_repu & 397 & 2.94 & .038 & .748 \\
\hline Prior_school_repu & 397 & 3.19 & .032 & .642 \\
\hline Prior_diversity & 397 & 3.03 & .037 & .738 \\
\hline Prior_facilities & 397 & 3.29 & .030 & .596 \\
\hline Prior_stay & 397 & 3.17 & .030 & .601 \\
\hline Prior_safe & 397 & 3.48 & .030 & .601 \\
\hline
\end{tabular}




\begin{tabular}{l|l|l|l|l|}
\hline Prior_med & 397 & 3.19 & .035 & .695 \\
\hline Prior_climate & 397 & 2.75 & .037 & .733 \\
\hline Prior_partner & 397 & 1.89 & .041 & .817 \\
\hline Prior_hybrid & 397 & 2.74 & .040 & .807 \\
\hline Cur_living & 397 & 3.45 & .030 & .595 \\
\hline Cur_travel & 397 & 2.33 & .041 & .817 \\
\hline Cur_language & 397 & 3.19 & .037 & .729 \\
\hline Cur_quality & 397 & 3.55 & .030 & .607 \\
\hline Cur_degree & 397 & 3.36 & .034 & .685 \\
\hline Cur_IntlStu & 397 & 2.73 & .042 & .830 \\
\hline Cur_VnStu & 397 & 2.44 & .043 & .853 \\
\hline Cur_tuition & 397 & 3.53 & .032 & .645 \\
\hline Cur_scholarship & 397 & 3.66 & .030 & .602 \\
\hline Cur_livingcost & 397 & 3.54 & .032 & .629 \\
\hline Cur_parttimejob & 397 & 3.20 & .039 & .783 \\
\hline Cur_job & 397 & 3.46 & .036 & .712 \\
\hline Cur_immigration & 397 & 2.77 & .047 & .931 \\
\hline Cur_shortcut & 397 & 2.68 & .044 & .869 \\
\hline Cur_distance & 397 & 2.31 & .046 & .912 \\
\hline Cur_HE_repu & 397 & 3.04 & .038 & .754 \\
\hline Cur_school_repu & 397 & 3.23 & .033 & .667 \\
\hline Cur_diversity & 397 & 3.08 & .036 & .712 \\
\hline Cur_facilities & 397 & 3.31 & .030 & .606 \\
\hline Cur_stay & 397 & 3.27 & .031 & .619 \\
\hline Cur_safe & 397 & 3.52 & .031 & .626 \\
\hline Cur_med & 397 & 3.39 & .034 & .671 \\
\hline Cur_climate & 397 & 2.88 & .041 & .824 \\
\hline Cur_partner & 397 & 1.84 & .041 & .823 \\
\hline Cur_hybrid & 397 & 3.01 & .040 & .799 \\
\hline & & & & \\
\hline
\end{tabular}

Table 3. Paired samples statistics Mean N Std. Deviation Std. Error Mean

\begin{tabular}{lll|l|l|l}
\hline Pair 1 & Prior_living & 3.34 & 397 & .566 & .028 \\
\cline { 2 - 7 } & Cur_living & 3.45 & 397 & .595 & .030 \\
\hline Pair 2 & Prior_travel & 2.46 & 397 & .690 & .035 \\
\cline { 2 - 6 } & Cur_travel & 2.33 & 397 & .817 & .041 \\
\hline \multirow{2}{*}{ Pair 3 } & Prior_language & 3.25 & 397 & .695 & .035 \\
\cline { 2 - 6 } & Cur_language & 3.19 & 397 & .729 & .037 \\
\hline Pair 4 & Prior_quality & 3.67 & 397 & .536 & .027 \\
\cline { 2 - 6 } & Cur_quality & 3.55 & 397 & .607 & .030 \\
\hline
\end{tabular}




\begin{tabular}{|c|c|c|c|c|c|}
\hline \multirow[t]{2}{*}{ Pair 5} & Prior_degree & 3.41 & 397 & .632 & .032 \\
\hline & Cur_degree & 3.36 & 397 & .685 & .034 \\
\hline \multirow[t]{2}{*}{ Pair 6} & Prior_IntlStu & 2.73 & 397 & .804 & .040 \\
\hline & Cur_IntlStu & 2.73 & 397 & .830 & .042 \\
\hline \multirow[t]{2}{*}{ Pair 7} & Prior_VnStu & 2.33 & 397 & .755 & .038 \\
\hline & Cur_VnStu & 2.44 & 397 & .853 & .043 \\
\hline \multirow[t]{2}{*}{ Pair 8} & Prior_tuition & 3.52 & 397 & .684 & .034 \\
\hline & Cur_tuition & 3.53 & 397 & .645 & .032 \\
\hline \multirow[t]{2}{*}{ Pair 9} & Prior_scholarship & 3.68 & 397 & .583 & .029 \\
\hline & Cur_scholarship & 3.66 & 397 & .602 & .030 \\
\hline \multirow[t]{2}{*}{ Pair 10} & Prior_livingcost & 3.54 & 397 & .617 & .031 \\
\hline & Cur_livingcost & 3.54 & 397 & .629 & .032 \\
\hline \multirow[t]{2}{*}{ Pair 11} & Prior_parttimejob & 3.20 & 397 & .739 & .037 \\
\hline & Cur_parttimejob & 3.20 & 397 & .783 & .039 \\
\hline \multirow[t]{2}{*}{ Pair 12} & Prior_job & 3.55 & 397 & .655 & .033 \\
\hline & Cur_job & 3.46 & 397 & .712 & .036 \\
\hline \multirow[t]{2}{*}{ Pair 13} & Prior_immigration & 2.78 & 397 & .881 & .044 \\
\hline & Cur_immigration & 2.77 & 397 & .931 & .047 \\
\hline \multirow[t]{2}{*}{ Pair 14} & Prior_shortcut & 2.58 & 397 & .845 & .042 \\
\hline & Cur_shortcut & 2.68 & 397 & .869 & .044 \\
\hline \multirow[t]{2}{*}{ Pair 15} & Prior_distance & 2.12 & 397 & .823 & .041 \\
\hline & Cur_distance & 2.31 & 397 & .912 & .046 \\
\hline \multirow[t]{2}{*}{ Pair 16} & Prior_HE_repu & 2.94 & 397 & .748 & .038 \\
\hline & Cur_HE_repu & 3.04 & 397 & .754 & .038 \\
\hline \multirow[t]{2}{*}{ Pair 17} & Prior_school_repu & 3.19 & 397 & .642 & .032 \\
\hline & Cur_school_repu & 3.23 & 397 & .667 & .033 \\
\hline \multirow[t]{2}{*}{ Pair 18} & Prior_diversity & 3.03 & 397 & .738 & .037 \\
\hline & Cur_diversity & 3.08 & 397 & .712 & .036 \\
\hline \multirow[t]{2}{*}{ Pair 19} & Prior_facilities & 3.29 & 397 & .596 & .030 \\
\hline & Cur_facilities & 3.31 & 397 & .606 & .030 \\
\hline \multirow[t]{2}{*}{ Pair 20} & Prior_stay & 3.17 & 397 & .601 & .030 \\
\hline & Cur_stay & 3.27 & 397 & .619 & .031 \\
\hline \multirow[t]{2}{*}{ Pair 21} & Prior_safe & 3.48 & 397 & .601 & .030 \\
\hline & Cur_safe & 3.52 & 397 & .626 & .031 \\
\hline \multirow[t]{2}{*}{ Pair 22} & Prior_med & 3.19 & 397 & .695 & .035 \\
\hline & Cur_med & 3.39 & 397 & .671 & .034 \\
\hline \multirow[t]{2}{*}{ Pair 23} & Prior_climate & 2.75 & 397 & .733 & .037 \\
\hline & Cur_climate & 2.88 & 397 & .824 & .041 \\
\hline Pair 24 & Prior_partner & 1.89 & 397 & .817 & .041 \\
\hline
\end{tabular}




\begin{tabular}{lll|l|l|l|}
\hline & Cur_partner & 1.84 & 397 & .823 & .041 \\
\hline Pair 25 & Prior_hybrid & 2.74 & 397 & .807 & .040 \\
\cline { 2 - 6 } & Cur_hybrid & 3.01 & 397 & .799 & .040 \\
\hline
\end{tabular}

Table 4. Paired samples correlations

\begin{tabular}{|c|c|c|c|c|}
\hline & & $\mathrm{N}$ & Correlation & Sig. \\
\hline Pair 1 & Prior_living \& Cur_living & 397 & .557 & .000 \\
\hline Pair 2 & Prior_travel \& Cur_travel & 397 & .667 & .000 \\
\hline Pair 3 & Prior_language \& Cur_language & 397 & .734 & .000 \\
\hline Pair 4 & Prior_quality \& Cur_quality & 397 & .586 & .000 \\
\hline Pair 5 & Prior_degree \& Cur_degree & 397 & .665 & .000 \\
\hline Pair 6 & Prior_IntlStu \& Cur_IntlStu & 397 & .634 & .000 \\
\hline Pair 7 & Prior_VnStu \& Cur_VnStu & 397 & .596 & .000 \\
\hline Pair 8 & Prior_tuition \& Cur_tuition & 397 & .636 & .000 \\
\hline Pair 9 & Prior_scholarship \& Cur_scholarship & 397 & .681 & .000 \\
\hline Pair 10 & Prior_livingcost \& Cur_livingcost & 397 & .649 & .000 \\
\hline Pair 11 & Prior_parttimejob \& Cur_parttimejob & 397 & .716 & .000 \\
\hline Pair 12 & Prior_job \& Cur_job & 397 & .648 & .000 \\
\hline Pair 13 & Prior_immigration \& Cur_immigration & 397 & .726 & .000 \\
\hline Pair 14 & Prior_shortcut \& Cur_shortcut & 397 & .648 & .000 \\
\hline Pair 15 & Prior_distance \& Cur_distance & 397 & .606 & .000 \\
\hline Pair 16 & Prior_HE_repu \& Cur_HE_repu & 397 & .640 & .000 \\
\hline Pair 17 & Prior_school_repu \& Cur_school_repu & 397 & .640 & .000 \\
\hline Pair 18 & Prior_diversity \& Cur_diversity & 397 & .664 & .000 \\
\hline Pair 19 & Prior_facilities \& Cur_facilities & 397 & .588 & .000 \\
\hline Pair 20 & Prior_stay \& Cur_stay & 397 & .552 & .000 \\
\hline Pair 21 & Prior_safe \& Cur_safe & 397 & .563 & .000 \\
\hline Pair 22 & Prior_med \& Cur_med & 397 & .522 & .000 \\
\hline Pair 23 & Prior_climate \& Cur_climate & 397 & .654 & .000 \\
\hline Pair 24 & Prior_partner \& Cur_partner & 397 & .722 & .000 \\
\hline Pair 25 & Prior_hybrid \& Cur_hybrid & 397 & .543 & .000 \\
\hline
\end{tabular}

Table 5. Paired samples test

\begin{tabular}{|c|c|c|c|c|c|c|c|c|c|}
\hline & \multicolumn{5}{|c|}{ Paired Differences } & \multirow[b]{3}{*}{$\mathrm{t}$} & \multirow[b]{3}{*}{$\mathrm{df}$} & \multirow{3}{*}{$\begin{array}{l}\text { Sig. (2- } \\
\text { tailed) }\end{array}$} \\
\hline & & \multirow[b]{2}{*}{ Mean } & \multirow[b]{2}{*}{ S.D } & \multirow{2}{*}{$\begin{array}{l}\text { S.E } \\
\text { Mean }\end{array}$} & \multicolumn{2}{|c|}{$\begin{array}{l}95 \% \text { Confidence } \\
\text { Interval of the } \\
\text { Difference }\end{array}$} & & & \\
\hline & & & & & Lower & Upper & & & \\
\hline $\begin{array}{l}\text { Pair } \\
1\end{array}$ & $\begin{array}{l}\text { Prior_living - } \\
\text { Cur_living }\end{array}$ & -.116 & .547 & .027 & -.170 & -.062 & $4.21 \overline{-}$ & 396 & .000 \\
\hline
\end{tabular}




\begin{tabular}{|c|c|c|c|c|c|c|c|c|c|}
\hline $\begin{array}{l}\text { Pair } \\
2\end{array}$ & $\begin{array}{l}\text { Prior_travel - } \\
\text { Cur_travel }\end{array}$ & .131 & .626 & .031 & .069 & .193 & 4.169 & 396 & .000 \\
\hline $\begin{array}{l}\text { Pair } \\
3\end{array}$ & $\begin{array}{l}\text { Prior_language - } \\
\text { Cur_language }\end{array}$ & .068 & .520 & .026 & .017 & .119 & 2.605 & 396 & .010 \\
\hline $\begin{array}{l}\text { Pair } \\
4\end{array}$ & $\begin{array}{l}\text { Prior_quality - } \\
\text { Cur_quality }\end{array}$ & .116 & .524 & .026 & .064 & .168 & 4.407 & 396 & .000 \\
\hline $\begin{array}{l}\text { Pair } \\
5\end{array}$ & $\begin{array}{l}\text { Prior_degree - } \\
\text { Cur_degree }\end{array}$ & .048 & .541 & .027 & -.006 & .101 & 1.761 & 396 & .079 \\
\hline $\begin{array}{l}\text { Pair } \\
6\end{array}$ & $\begin{array}{l}\text { Prior_IntlStu - } \\
\text { Cur_IntlStu }\end{array}$ & .005 & .700 & .035 & -.064 & .074 & .143 & 396 & .886 \\
\hline $\begin{array}{l}\text { Pair } \\
7\end{array}$ & $\begin{array}{l}\text { Prior_VnStu - } \\
\text { Cur_VnStu }\end{array}$ & -.113 & .728 & .037 & -.185 & -.042 & 3.102 & 396 & .002 \\
\hline $\begin{array}{l}\text { Pair } \\
8\end{array}$ & $\begin{array}{l}\text { Prior_tuition - } \\
\text { Cur_tuition }\end{array}$ & -.015 & .568 & .029 & -.071 & .041 & -.530 & 396 & .597 \\
\hline $\begin{array}{l}\text { Pair } \\
9\end{array}$ & $\begin{array}{l}\text { Prior_scholarship - } \\
\text { Cur_scholarship }\end{array}$ & .023 & .474 & .024 & -.024 & .069 & .954 & 396 & .341 \\
\hline $\begin{array}{l}\text { Pair } \\
10\end{array}$ & $\begin{array}{l}\text { Prior_livingcost - } \\
\text { Cur_livingcost }\end{array}$ & .000 & .522 & .026 & -.052 & .052 & .000 & 396 & 1.000 \\
\hline $\begin{array}{l}\text { Pair } \\
11\end{array}$ & $\begin{array}{l}\text { Prior_parttimejob - } \\
\text { Cur_parttimejob }\end{array}$ & .008 & .575 & .029 & -.049 & .064 & .262 & 396 & .794 \\
\hline $\begin{array}{l}\text { Pair } \\
12\end{array}$ & Prior_job - Cur_job & .098 & .576 & .029 & .041 & .155 & 3.401 & 396 & .001 \\
\hline $\begin{array}{l}\text { Pair } \\
13\end{array}$ & $\begin{array}{l}\text { Prior_immigration - } \\
\text { Cur_immigration }\end{array}$ & .018 & .672 & .034 & -.049 & .084 & .523 & 396 & .601 \\
\hline $\begin{array}{l}\text { Pair } \\
14\end{array}$ & $\begin{array}{l}\text { Prior_shortcut - } \\
\text { Cur_shortcut }\end{array}$ & -.098 & .720 & .036 & -.169 & -.027 & 2.719 & 396 & .007 \\
\hline $\begin{array}{l}\text { Pair } \\
15\end{array}$ & $\begin{array}{l}\text { Prior_distance - } \\
\text { Cur_distance }\end{array}$ & -.191 & .774 & .039 & -.268 & -.115 & 4.926 & 396 & .000 \\
\hline $\begin{array}{l}\text { Pair } \\
16\end{array}$ & $\begin{array}{l}\text { Prior_HE_repu - } \\
\text { Cur_HE_repu }\end{array}$ & -.103 & .637 & .032 & -.166 & -.040 & $3.230^{-}$ & 396 & .001 \\
\hline $\begin{array}{l}\text { Pair } \\
17\end{array}$ & $\begin{array}{l}\text { Prior_school_repu - } \\
\text { Cur_school_repu }\end{array}$ & -.038 & .556 & .028 & -.093 & .017 & $\begin{array}{r}- \\
1.354\end{array}$ & 396 & .177 \\
\hline $\begin{array}{l}\text { Pair } \\
18\end{array}$ & $\begin{array}{l}\text { Prior_diversity - } \\
\text { Cur_diversity }\end{array}$ & -.048 & .595 & .030 & -.107 & .011 & 1.603 & 396 & .110 \\
\hline $\begin{array}{l}\text { Pair } \\
19\end{array}$ & $\begin{array}{l}\text { Prior_facilities - } \\
\text { Cur_facilities }\end{array}$ & -.020 & .546 & .027 & -.074 & .034 & -.736 & 396 & .462 \\
\hline $\begin{array}{l}\text { Pair } \\
20\end{array}$ & Prior_stay - Cur_stay & -.101 & .577 & .029 & -.158 & -.044 & $\begin{array}{r}- \\
3.478\end{array}$ & 396 & .001 \\
\hline $\begin{array}{l}\text { Pair } \\
21\end{array}$ & Prior_safe - Cur_safe & -.038 & .574 & .029 & -.094 & .019 & $1.312^{-}$ & 396 & .190 \\
\hline $\begin{array}{l}\text { Pair } \\
22\end{array}$ & Prior_med - Cur_med & -.196 & .668 & .034 & -.262 & -.131 & 5.861 & 396 & .000 \\
\hline
\end{tabular}




\begin{tabular}{llr|r|r|r|r|r|r|r}
\hline Pair & $\begin{array}{l}\text { Prior_climate - } \\
23\end{array}$ Cur_climate & -.136 & .653 & .033 & -.200 & -.072 & -396 & .000 \\
\hline $\begin{array}{l}\text { Pair } \\
24\end{array}$ Prior_partner - & Cur_partner & .048 & .612 & .031 & -.012 & .108 & 1.559 & 396 & .120 \\
\hline $\begin{array}{l}\text { Pair } \\
25\end{array}$ & $\begin{array}{l}\text { Prior_hybrid - } \\
\text { Cur_hybrid }\end{array}$ & -.264 & .767 & .039 & -.340 & -.189 & -396 & .000 \\
\hline
\end{tabular}

\section{Experimental Design, Materials, and Methods}

The research group collected data with snowball and convenience sampling methods. The survey was published on the biggest Facebook groups of current and potential Vietnamese overseas students (Scholarship hunter) and then was spread wider by those group members. From 03 May 2020 to 13 May May 2020, a total of 410 responses was collected. After cleaning the dataset, 13 invalid observations were cleared, we finally analyzed the dataset of 397 observations.

Since shorter scales are "quick to use" [12], the research group used a four-point Linkert scale to reach a specific respondent opinion and avoid "neutral" responses as well.

Table 1 shows descriptive statistics about participants' demographics including gender, major, current location, financial source, and study program. Table 2 shows descriptive information regarding influence factors of study abroad decision before the COVID-19 (Prior-) and during the pandemic (Cur-). Those 25 factors are classified into six categories according to Lee's model of decision-making process of studying abroad [13]. This model is the integration of three-phase synthesis model [14] and push-pull model [15].

Lee's model [11] originally includes seven dimensions: (i) Personal improvement (Prior_personal, Cur_personal), (ii) Knowledge and awareness of the host country (Prior_knowledge, Cur_knowledge), (iii) Recommendation from others, (iv) Physical and learning environment (Prior_learning, Cur_learning), (v) Cost issues (Prior_cost, Cur_cost), (vi) Social links and geographic proximity (Prior_social, Cur_social), and (vii) Institution image (Prior_institution, Cur_institution). However, in order to adapt better to Vietnam context, researchers dropped dimension (iii) as well as omitted and modified some factors within other dimensions. Table 3, 4 and 5 illustrates results of t-test between influence factors before and during the COVID-19.

Moreover, the dataset includes destination countries where students plan to go to, which can be divided into five categories: Asia, Europe, America, Oceania and Other [16]. As a consequence, future researchers can study on the crucial factors that determine destination choice of overseas students, and whether those factors play different roles before and during the pandemic:

Prior_Plan $\sim \beta 0+\beta 1 *($ Prior_personal $)+\beta 2 *($ Prior_knowledge $)+\beta 3 *($ Prior_learning $)+$ $\beta 4 *($ Prior_cost $)+\beta 5^{*}($ Prior_social $)+\beta 6^{*}($ Prior_institution $)+\mathrm{u}$

Cur_Plan $\sim \beta 0+\beta 1 *$ (Cur_personal) $+\beta 2 *($ Cur_knowledge $)+\beta 3 *($ Cur_learning $)+$ $\beta 4 *($ Cur_cost $)+\beta 5^{*}($ Cur_social $)+\beta 6^{*}($ Cur_institution $)+\mathrm{u}$

In addition, the change in decision to study abroad (AFFECT) was showed by question number 8 (from 8.1 to 8.5 ), thus future study can use multinomial logistic regression to find the relationship 
between various factors (before and during the COVID-19) and change in the decision to study abroad as the following model:

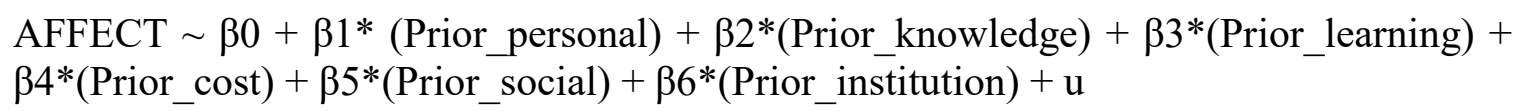

\section{Acknowledgments}

We would like to thank all students who helped us to finish this study, as well as admins of various Facebook communities, teachers and school leaders, who contributed to elevating the data collection process.

This research is funded by Vietnam's National Foundation for Science and Technology Development (NAFOSTED) under the project 502.02-2019.22

\section{Competing Interests}

The authors declare that they have no known competing financial interests or personal relationships which have, or could be perceived to have, influenced the work reported in this article.

\section{References}

[1] Dwyer, M. M., \& Peters, C. K. (2004). The benefits of study abroad. Transitions abroad, 37(5), 56-58.

[2] Altbach, P. G., \& Knight, J. (2007). The internationalization of higher education: Motivations and realities. Journal of studies in international education, 11(3-4), 290-305.

[3] Healey, N. M. (2008). Is higher education in really 'internationalising'? Higher Education, $55,333-355$.

[4] Pham, H. H., Lai, S. L., \& Vuong, Q. H. (2019). The Role of Subjective Task Value in Forming Satisfaction and Loyalty Among Vietnamese International Students: A Structural Equation Model. The Asia-Pacific Education Researcher, 28(5), 399-409.

[5] Open Doors. (2019). 2019 Fast facts. U.S. The Institute of International Education. https://www.iie.org/en/Research-and-Insights/Open-Doors/Fact-Sheets-and-Infographics/Fast$\underline{\text { Facts }}$

[6] Bich Tram. (2019). Chi 3 tỉ USD mỗi năm, du học sinh Việt Nam đến nước nào? [Spending \$3 billion per year, where do Vietnamese students go to study abroad?]. Forbes Vietnam. https://forbesvietnam.com.vn/tin-cap-nhat/chi-3-ti-usd-moi-nam-du-hoc-sinh-viet-nam-dennuoc-nao-8274.html 
[7] Coronavirus: Travel restrictions, border shutdowns by country. (2020). Al Jazeera. https://www.aljazeera.com/news/2020/03/coronavirus-travel-restrictions-border-shutdownscountry-200318091505922.html

[8] Hoang, Anh-Duc, 2020, "Survey on ex-pat teachers' intention to leave due to COVID-19", [dataset] https://doi.org/10.7910/DVN/ZB2DNH, Harvard Dataverse, V1.

[9] Hoang, A. D., Ta, N. T., Nguyen, Y. C., Hoang, C. K., Nguyen, T. T., Pham, H. H., ... \& Dinh, V. H. (2020). Dataset of Ex-pat Teachers in Southeast Asia's Intention to Leave due to the COVID-19 pandemic. Data in Brief, 105913.

[10] Tran, T., Hoang, A. D., Nguyen, Y. C., Nguyen, L. C., Ta, N. T., Pham, Q. H., Pham, C.X., Le, Q. A., Dinh, V. H., Nguyen, T. T. (2020). Toward Sustainable Learning during School Suspension: Socioeconomic, Occupational Aspirations, and Learning Behavior of Vietnamese Students during COVID-19. Sustainability, 12(10):4195. https://doi.org/10.3390/su12104195

[11] Pham, H.H., Phan, Q.A., Hoang, A.D., Nguyen, Y.C., Ta, N.T., Dinh, V.H. (2020). "Survey on Vietnamese students' intention to study abroad before and during COVID-19", Mendeley Data, v1http://dx.doi.org/10.17632/7gpkx5p3z9.1

[12] Preston, C. C., \& Colman, A. M. (2000). Optimal number of response categories in rating scales: reliability, validity, discriminating power, and respondent preferences. Acta psychologica, 104(1), 1-15. doi:10.1016/S0001-6918(99)00050-5.

[13] Lee, C. F. (2014). An investigation of factors determining the study abroad destination choice: A case study of Taiwan. Journal of Studies in International Education, 18(4), 362-381.

[14] Chen, L. H. (2007). East-Asian students' choice of Canadian graduate schools. International Journal of Educational Advancement, 7(4), 271-306

[15] Mazzarol, T., \& Soutar, G. N. (2002). "Push-pull" factors influencing international student destination choice. International Journal of Educational Management, 16(2), 82-90.

[16] Open Doors. (2019). Data by Region Fact Sheets. U.S. The Institute of International Education. https://www.iie.org/Research-and-Insights/Open-Doors/Fact-Sheets-andInfographics/Data-by-Region-Fact-Sheets 